\title{
Idiopathic Chylothorax in Infants - Is Octreotide Alone Enough?
}

\author{
Maralihalli $\mathrm{MB}^{1}$, Matti $\mathrm{MR}^{2}$, Joshi $\mathrm{SN}^{3}$, Annigeri $\mathrm{VM}^{4}$
}

\begin{abstract}
Five month old female baby presented to us for evaluation of asymptomatic right sided pleural effusion. Pleural fluid analysis revealed it as chylous effusion. Radiological investigations revealed gross right side pleural effusion with partial collapse of right lung. After investigations, diagnosis of asymptomatic idiopathic chylothorax was made. Baby was treated with Inj. Octreotide alone. Pleural drain was not placed. After three doses of Octreotide, there was complete clearance of pleural effusion.
\end{abstract}

Key words: Asymptomatic pleural effusion, Chylothorax, Octreotide.

\section{Introduction}

C hylothorax occurs due to accumulation of lymphatic fluid in the pleural cavity. It can occur spontaneously in the neonates or due to developmental anomalies of lymphatic, or following injury to thoracic duct or lymphatic vessels. In most cases its difficult to establish etiology, particularly with congenital lesions. Loss of lymphatic fluid into pleural cavity can cause nutritional, electrolyte, and immunologic complications. Diagnosis is typically established by its clinical and biochemical characteristics including triglycerides content of $1.1 \mathrm{mmol} / \mathrm{L}$ or more and a total cell count of 1000 cells/ microlitre or more with $80 \%$ or more lymphocytes ${ }^{1}$. There are no specific guidelines about the treatment for idiopathic congenital chylothorax. Most of the children respond to conservative measures in non-iatrogenic cases. Most advice, adequate drainage of the pleural fluid, along with changes in enteral feeds. Recently Octreotide has been used successfully in treatment of congenital chylothorax in few cases. Here we present a five month baby who responded to only three doses of Octreotide alone.

\section{The Case}

Five month old female baby was referred to us with a chest $x$-ray showing right sided pleural effusion, that was taken during an episode of acute illness. Baby was delivered by cesarean section, cried after birth. Infant was gaining weight adequately. On admission, baby had stable vitals. Her weight and height were normal for age. There were no pallor, edema, hepatosplenomegaly, icterus, lymphadenopathy.
'Dr. Mahesh B Maralihalli, MBBS, MD, Assistant Professor, ${ }^{2}$ Dr. Mahantesh R Matti, MBBS, MD, ${ }^{3}$ Dr. Suhas N Joshi, MBBS, MD. All from Department of Paediatrics, SDM College of Medical Sciences and Hospital Sattur, Dharwar Karnataka. ${ }^{4}$ Dr. Venkatesh M Annigeri MBBS, MS, MCh. Department of Paediatric Surgery, SDM College of Medical Sciences and Hospital Sattur, Dharwar Karnataka, India.

\section{Address for correspondence:}

Dr. Mahesh Maralihalli

E-mail: drmaheshmaralihalli@gmail.com

Maralihalli MB, Matti MR, Joshi SN, Annigeri VM. Idiopathic Chylothorax in Infants - Is Octreotide Alone Enough? J Nepal Paediatr Soc 2015;35(1):6466.

doi: http://dx.doi.org/10.3126/jnps.v35i1.11855

This work is licensed under a Creative Commons Attribution 3.0 License.

\section{(c) (i)}

General physical examination was normal. There was decreased air entry in right lung field. Other systems were normal. There were no dysmorphic features. Her blood counts, ESR, C-reactive protein, electrolytes, urea and creatinine were normal. CT chest (Figure 1) showed gross right pleural effusion with partial collapse of right lung, mediastinal shift towards left side.

Pleural tap fluid was milky, opalescent on appearance. Pleural fluid analysis showed predominant lymphocytes with occasional mesothelial cells, Triglycerides 5154 mg\%, proteins 5.6 gm\%, sugar 212 
mg\% suggestive of chylous effusion, ZN stain was negative for Acid fast bacilli, pleural fluid culture was sterile. Octreotide was given as intravenous infusion at dosage of $100 \mu \mathrm{g} / \mathrm{kg}$ over 10 hours $(10 \mu \mathrm{g} / \mathrm{kg} /$ hour) once a day. Baby's vitals, blood sugar, cardiac monitoring was done. After three days of treatment chest $X$ ray (Figure 2 ) was taken, showing complete clearance pleural effusion. A chest $X$ ray was taken after 15 days of follow up, was normal.

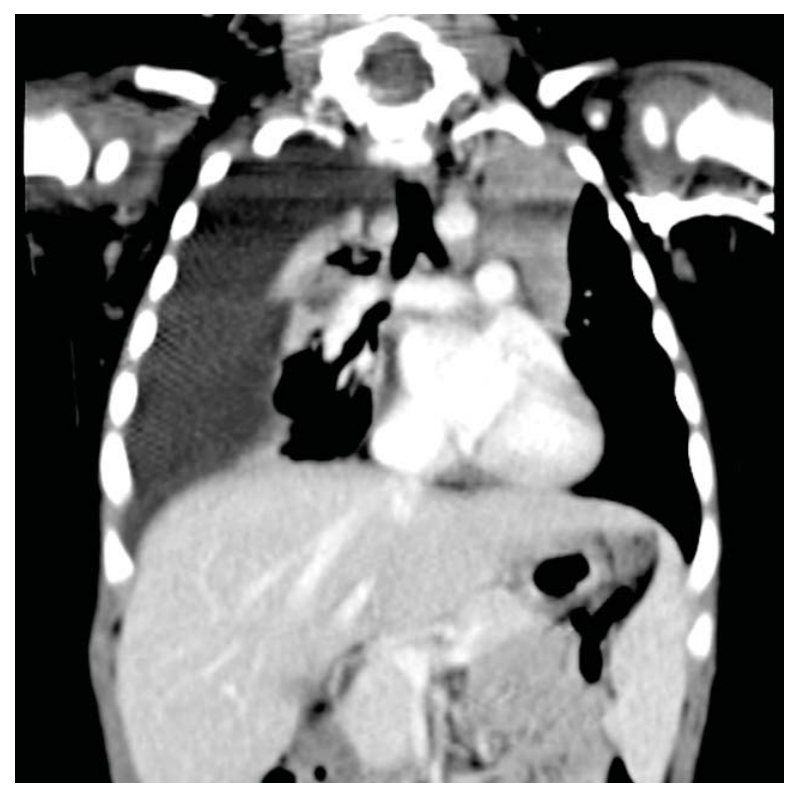

Fig 1: CT Chest of the baby showing right sided pleural effusion

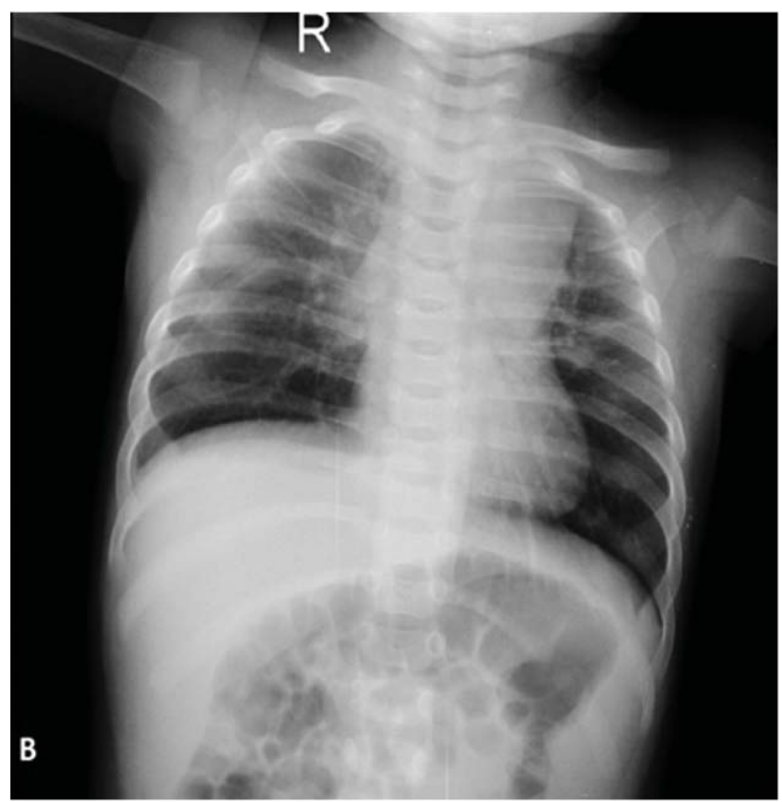

Fig 2: Chest X-ray taken after three doses of Octreotide therapy showing resolution of effusion.

\section{Discussion}

Congenital or primary chylothorax is the most common type of pleural effusion seen in fetuses and newborns, $50 \%$ of chylothoraces are present at birth or appear during the first week of life. It may be associated with Down syndrome, Noonan or Turner syndrome. Other etiologies are classified as traumatic and non traumatic ${ }^{2}$. Traumatic causes include Noniatrogenic (childbirth, blunt trauma to chest) and iatrogenic causes (thoracic surgery, head and neck surgery). Non-traumatic causes include malignancy, diseases (Tuberculosis, Benign tumor) and Idiopathic. Most cases of neonatal chylothorax occur without clear etiology, termed as idiopathic congenital chylothorax.

This infant was incidentally found to have pleural effusion. Diagnostic pleural tap showed chylous fluid. CT chest showed gross right side pleural effusion. There was no lymphadenopathy, left lung was normal. Investigations showed no evidence to the possible etiology. Lymphangiography could not be done due to non availability at our centre.

Optimal treatment guidelines remain debatable. Conservative treatment includes use of a low-fat diet supplemented with medium chain triglycerides or total parenteral nutrition (TPN). This option requires placement of pleural drain. Surgical intervention is warranted if pleural drainage persists beyond 2 to 5 weeks of TPN. Surgical options are thoracic duct ligation, pleuroperitonial shunting, pleurectomy, or pleurodesis $^{1}$. Few case reports have been published about the successful use of Octreotide, a somatostatin anologue in congenital or postoperative chylothorax in children ${ }^{3,4}$. Octreotide may be given subcutaneously (20-70 $\mathrm{\mu g} / \mathrm{kg} / \mathrm{day}, \mathrm{TDS})$, or as an intravenous infusion $(1-10 \mu \mathrm{g} / \mathrm{kg} / \mathrm{hr})$. The duration of therapy depends on the reduction in pleural drain volume ${ }^{5}$. Octreotide therapy durations were longer while treating congenital chylothoraces than in postoperative cases, ranging from 3 to 29 days $^{3}$. Octreotide is believed to act by decreasing lymphatic flow through a reduction in gastric, intestinal, and pancreatic secretions or by a decrease in hepatic venous pressure and splanchnic blood flow. Octreotide is used for the treatment of growth hormone producing tumors, diarrhea and flushing episodes associated with carcinoid syndrome and diarrhea associated with vasoactive intestinal peptide secreting tumors. Adverse effects are headache, cardiac conduction changes, vomiting, hyper or hypoglycemia.

This infant had no respiratory distress and was on exclusive breast feeding. Pleural drain was not placed. 
There was complete clearance of pleural effusion after three doses of Octreotide. We believe this is one of the few cases where in only Octreotide has been used without pleural drain to treat idiopathic asymptomatic chylothorax and complete clearance of effusion as early as with three doses only. But more studies and evidences are required before selection of cases for Octreotide alone therapy.

\section{Conclusion}

There are no optimal guidelines available for treatment of idiopathic chylothorax in infants. Few cases have been treated with Octreotide after surgical drainage. But in our case, only Octreotide was used to treat and complete clearance of the pleural effusion was documented as early as 3days only. We believe that this is one of the very few cases wherein only Octreotide has been used to treat idiopathic chylothorax and to document complete clearance of chylothorax within three days only.

\section{References}

1. Beghetti M, La Scala G, Belli D, et al. Etiology and management of pediatric chylothorax. J Pediatr 2000;136(5):3.

2. Nair SK, Petko M, Hayward MP. Aetiology and management of chylothorax in adults. Eur J Cardiothoracic Surg 2007;32(2):362-9.

3. Coulter DM. Successful treatment with octreotide of spontaneous chylothorax in a premature infant. J Perinatol 2004;24:194-195.

4. Sivasli E, Dogru D, Aslan AT, Yurdakok M, Tekinalp G. Spontaneous neonatal chylothorax treated with octreotide in Turkey: a case report. J Perinatol 2004;24:261-262.

5. Buck ML. Octreotide for the management of chylothorax in infants and children. Pediatr Pharm 2004;10(10). 